\title{
Effects of environment, dietary regime and ageing on the dengue vector microbiota: evidence of a core microbiota throughout Aedes aegypti lifespan
}

\author{
Mariana Rocha David', Lilha Maria Barbosa dos Santos', \\ Ana Carolina Paulo Vicente ${ }^{2}$, Rafael Maciel-de-Freitas ${ }^{1 /+}$
}

Fundação Oswaldo Cruz, Instituto Oswaldo Cruz, Laboratório de Mosquitos Transmissores de Hematozoários, Rio de Janeiro, RJ, Brasil

${ }^{2}$ Fundação Oswaldo Cruz, Instituto Oswaldo Cruz, Laboratório de Genética Molecular de Microrganismos, Rio de Janeiro, RJ, Brasil

Mosquito midgut microbiota is a key component of vector competence, as gut bacteria can disturb pathogen development. In this study, we addressed the microbiota composition of Aedes aegypti during its lifespan, under field conditions. We also investigated the possible effects of environment, dietary regime and ageing on the gut community composition. We employed culture independent and dependent approaches to characterise vector microbiota. There was evidence of a lifelong stable core microbiota after mosquitoes were released into an urban settlement, where they presumably fed on a range of vertebrate hosts and carbohydrate sources. This core was formed mainly of bacteria belonging to the genera Pseudomonas, Acinetobacter, Aeromonas and Stenotrophomonas and to the families Oxalobacteraceae, Enterobacteriaceae and Comamonadaceae. We showed that both dietary regime and age were associated with the abundance of some bacterial groups in the Ae. aegypti microbiota. The majority of the bacterial groups we identified have been detected in the midgut of Ae. aegypti from laboratory and wild populations, indicating a possible core microbiota associated with this mosquito species. Our findings suggest that Ae. aegypti harbours a stable bacterial community during its adult life, similar to mosquito populations from distinct geographic areas, which may be further explored for arbovirus biocontrol strategies.

Key words: Aedes - microbiota - diversity - mark-release-recapture

The microbial communities colonising cells and organs of higher organisms (microbiota) interact with hosts in a continuum of ecological interactions ranging from parasitism to mutualism. The microbiota is believed to provide the host with valuable capabilities, which influence physiology and improve fitness (Rosenberg \& Rosenberg 2008). The insect digestive tract harbours microbial communities, comprised of viruses, bacteria, yeasts and protists. Insect vector bacterial microbiota, especially of mosquitoes, is known to play an important role in host nutrition, reproduction, development, immunity and protection against pathogens (Minard et al. 2013).

Recent reports have demonstrated that mosquito midgut microbiota is a key component of vector competence, as gut bacteria can inhibit the development of pathogens, including Plasmodium and dengue virus (DENV) (Xi et al. 2008, Dong et al. 2009, Ramírez et al. 2012, 2014). These findings suggest the introduction of particular bacterial strains, harbouring innate or engineered antipathogen activity, into the mosquito gut as a novel disease control strategy (Gusmão et al. 2010, Ramírez et al. 2012,

doi: 10.1590/0074-02760160238

Financial support: CNPq, FAPERJ, FIOCRUZ, CAPES.

+ Corresponding author: freitas@ioc.fiocruz.br

Received 29 May 2016

Accepted 27 June 2016
2014, Villegas \& Pimenta 2014). For the effective application of this approach in the field, it is mandatory to know which factors influence the microbiota diversity of the target vector species, especially under natural conditions.

In the laboratory environment, dietary regime (Gusmão et al. 2010, Terenius et al. 2012) and chikungunya virus infection (Zouache et al. 2012) influenced the microbiota diversity of adult Aedes mosquitoes. In wild populations, the host genetic diversity (Minard et al. 2015) and habitat (Zouache et al. 2011, Osei-Poku et al. 2012, Kim et al. 2015) modulated the midgut community composition. In this scenario, the understanding of midgut bacterial community dynamics throughout the Aedes aegypti lifespan, under natural conditions, is still imperative.

The objective of this study was to profile the midgut microbial diversity throughout the Ae. aegypti lifespan and address possible determinants of microbiota structure in adult females. Since the environment is believed to influence midgut community composition in mosquitoes (Zouache et al. 2011, Osei-Poku et al. 2012, Kim et al. 2015), we investigated whether the release of laboratory reared females into the urban environment would result in changes in their microbiota composition over time. Therefore, we performed a mark-release-recapture experiment, a reliable tool for the ecological study of a target species in its natural habitat. We identified gut bacteria employing culture-independent (deep-sequencing) and -dependent approaches in female mosquitoes recaptured on different days after release. We also characterised the microbiota diversity according to diet (sugar or blood) and mosquito age in laboratory and wild insect populations. 


\section{MATERIALS AND METHODS}

Study area - The study was conducted in a section of 15 blocks in Vila Valqueire, a middle class suburban district located in the northeast of Rio de Janeiro city, RJ, Brazil $\left(22^{\circ} 53^{\prime} 09^{\prime \prime} \mathrm{S} ; 43^{\circ} 21^{\prime} 59^{\prime \prime} \mathrm{W}\right)$. It is a sparsely populated area surrounded by a secondary forest with paved streets, regular municipal water supply and garbage collection. The region contains 410 houses, a majority with three-four bedrooms and extended yards, with human density of 7,4 inhabitants $/ \mathrm{km}^{2}$. This neighbourhood was chosen for the mark-release-recapture experiment because it offered the highest mosquito collection rate, among the five locations in Rio de Janeiro monitored by our group, with dengue outbreaks occurring every three-four years. Weekly sampling in Vila Valqueire for two consecutive years revealed an average of 1.3 female mosquitoes per house per day. Water tanks and buckets have been indicated as the most productive Ae. aegypti breeding containers (Dutra et al. 2015).

Mark-release-recapture experiment - The Ae. aegypti mosquitoes (F1 generation) were derived from a laboratory colony established from eggs collected in Vila Valqueire with 40 ovitraps uniformly distributed throughout the neighbourhood, to collect a representative sample of the genetic variation in the population. Larvae for the experiment were fed commercial fish food $\left(\right.$ Tetramin $^{\circledR}$, Spectrum Brands, Madison, WI, USA). Adults were fed ad libitum $10 \%$ sucrose, until the release day. Males and females were maintained in the same cage for $72 \mathrm{~h}$ under laboratory conditions $(25 \pm$ $5^{\circ} \mathrm{C}$ and $60 \pm 8 \%$ relative humidity) to stimulate mating.

Mated and sugar-fed three-day-old females were marked with fluorescent dust (Day-Glo Color Corp., Cleveland, $\mathrm{OH}, \mathrm{USA}$ ) and then released outdoors at a local square, to ensure mosquito dispersal through the neighbourhood. Daily captures were performed with backpack aspirators (John W Hock, Gainesville, FL, USA), starting on the day following release. A total of 15 houses were randomly selected daily for insect aspiration for 15-20 min per house, including the peridomestic area. Mosquitoes were recaptured within a range of 0.5 $\mathrm{km}$, according to the short flight range usually observed for Ae. aegypti (McDonald 1977, Harrington et al. 2005, Maciel-de-Freitas et al. 2007).

The recaptured females were transported alive to the laboratory to preserve DNA quality, anaesthetised on ice, and checked for the fluorescent marking under UV light. Captures ended when no marked females were collected in three consecutive days. Fiocruz Ethical Committee approved the mark-release-recapture experiment in Vila Valqueire (CEP protocol number 22286313.7.0000.5248); permission was also obtained from the Rio de Janeiro Department of Health. In order to recapture marked mosquitoes inside dwellings, local residents received a full explanation of the project from at least one of the co-authors. In addition, mosquito collection is vector surveillance routine in Rio de Janeiro; therefore, many residents are used to receiving health agents in their houses. Collections were performed after obtaining formal written consents from the household- ers. There were no releases during dengue outbreaks or if dengue cases were reported in that neighbourhood.

Midgut microbiota samples - The diversity of midgut microbiota of Ae. aegypti females collected from the field was evaluated by $16 \mathrm{~s}$ rDNA gene pyrosequencing in (a) female mosquitoes released and recaptured (MRR) two, four, six, seven and eight days after release (DAR) and (b) wild females (WD). The profiling of wild insects, i.e. unmarked insects, was conducted to provide control for the effect of laboratory rearing on female gut microbiota. The majority of females recaptured during the mark-release-recapture assay $(81 \%)$ had blood content in the midgut, a condition for microbiota investigation. Bacterial isolation was performed for all groups, except WD (Table I). The likelihood of collecting naturally infected mosquitoes during an interepidemic period is low; therefore, we did not conduct any assay to test whether the captured mosquitoes were infected with DENV.

A portion of the mosquitoes from the same generation/batch not released in the natural environment was maintained in the laboratory, where we assessed the microbiota of (c) sugar-fed young (SFY), (d) blood-fed young (BFY), and (e) sugar-fed old (SFO) females (Table I). This aided in determining the effect of diet regime on microbiota composition before and after blood feeding. In addition, we seldom recaptured females $\sim 10$ days post release; therefore, we also sampled 38-day-old laboratory insects to evaluate the effect of ageing on microbiota diversity.

Midgut dissection - Only live insects were processed in order to preserve DNA quality for microbiota investigation. Ice-anaesthetised mosquitoes were surface-sterilised in $70 \%$ ethanol for $1 \mathrm{~min}$ and rinsed with sterile phosphate-buffered saline (PBS). Soon after surface sterilisation, mosquitoes were submerged in sterile PBS. An aliquot of this PBS was plated on Luria-Bertani agar media (LBA), which was kept at room temperature for seven days. This procedure was conducted to ensure mosquito external surface sterilisation. If bacterial growth occurred on the LBA, the corresponding samples were discarded, because they were potentially contaminated by bacteria from the external surface of the mosquitoes. Midguts were removed on a sterile glass slide and macerated in sterile PBS. Each sample consisted of two midguts. Females from the mark-release-recapture experiment were coupled according to their recapture day and the presence of blood in their intestinal tract.

16S rRNA library construction and 454 pyrosequencing - Total DNA from the midgut samples was extracted with the DNeasy Blood \& Tissue Kit (Qiagen, Redwood City, CA, USA). The hypervariable regions (V3 to V5) of bacterial 16S rRNA gene were amplified with 926R (5'-CCGTCAATTCMTTTRAGT-3') and 357F (5'-CCTACGGGAGGCAGCAG-3') primers containing 454 sequencing adapters and Multiplex Identifier (MID) tags, affording the multiplexing of samples (HMP Consortium, 2010). Polymerase chain reaction (PCR) was performed with Platinum Taq DNA Polymerase High Fidelity (Invitrogen, Carlsbad, CA, USA), with initial denaturation at $95^{\circ} \mathrm{C} / 2 \mathrm{~min}$ and 30 cycles of denatura- 


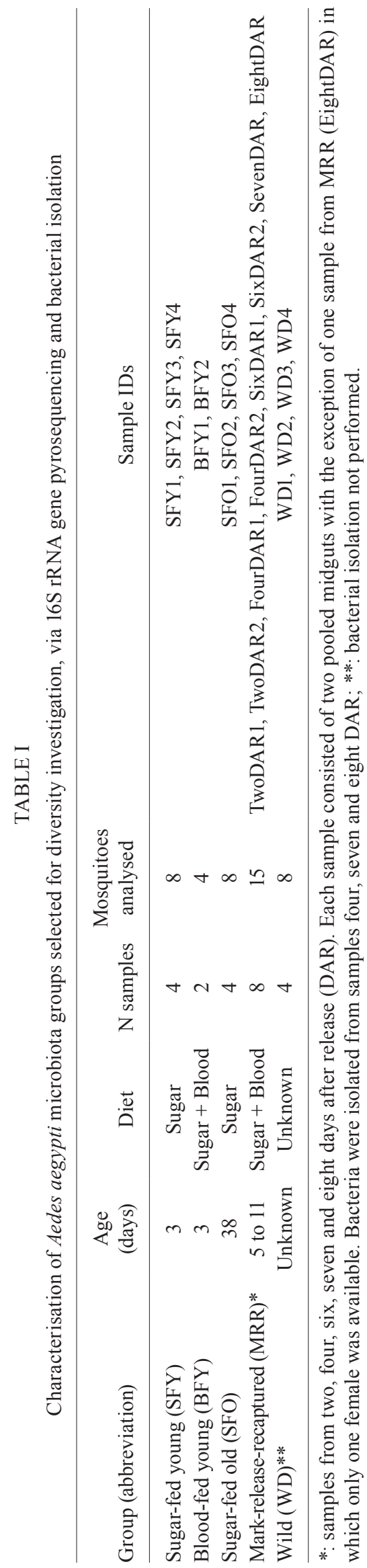

tion at $95^{\circ} \mathrm{C} / 20 \mathrm{sec}$, annealing at $50^{\circ} \mathrm{C} / 30 \mathrm{sec}$, and extension at $72^{\circ} \mathrm{C} / 5 \mathrm{~min}$ (HMP Consortium, 2010). Each $16 \mathrm{~S}$ rRNA amplicon library was constructed from three independent PCRs. PCR products were purified with the Agencourt AMPure XP kit (Beckman Coulter, Brea, CA, USA). Twenty-four libraries were constructed, 22 from midgut samples and two controls. Pyrosequencing was performed using a high-throughput platform/Fiocruz, with the 454 Genome Sequencer Junior System (Roche, Basel, Switzerland). Sequences were deposited in the National Center for Biotechnology Information Sequence Reads Archive (accession number SRR2916651).

Bioinformatics and operational taxonomic unit (OTU) assignment - Data were trimmed, filtered and analysed with the Mothur package v.1.31.2 (Schloss et al. 2009). Sequence reads were sorted into appropriate files according to MID tags, allowing two errors on the primer sequence and one on the barcode sequence. Additionally, sequences were trimmed to 450 flowgrams. After the trimming process, reads less than $200 \mathrm{bp}$ were discarded. Finally, potential chimeric sequences were removed with UCHIME, implemented on Mothur software (Edgar et al. 2011). All unique sequences were aligned to the SILVA-database reference alignment (release 119) and classified with the Mothur implementation of the naïve Bayesian classifier (Wang et al. 2007). Taxonomic assignment was based on the Ribosomal Data Project reference files (Training Set v.9) with an $80 \%$ bootstrap cut off. Pyrosequencing produced $\sim 360,000$ sequence reads from Ae. aegypti midgut samples. After primer and barcode removal, quality trimming, size filtering and chimera exclusion, 245,647 reads (241-307 bp long) were considered to be of good quality for taxonomic analysis.

Sequence reads with maximum $3 \%$ distance were clustered into OTUs. The OTUs represented by a single read or detected in control samples in abundance smaller than 10 times greater than in controls were discarded (Minard et al. 2015). Sample coverage was evaluated through rarefaction curves, which describe the number of OTUs detected as a function of sequencing generated reads. The sampling effort across samples was standardised by randomly selecting the smallest number of sequences among all the samples.

Diversity and statistical analysis - Alpha diversity was evaluated with OTU clustering through richness, abundance-based coverage estimator (ACE), Shannon index, inverse Simpson index and evenness. Diversity metrics were compared between groups with one-way analysis of variance (ANOVA; followed by Tukey's posthoc test) or Kruskal-Wallis test (followed by Dunn's test), depending on normal distribution verification with the Shapiro-Wilk test. P-values were adjusted for multiple comparisons by the Bonferroni criteria.

Principal coordinates analysis (PCoA) and non-parametric multivariate ANOVA (NPMANOVA or "adonis"), both with a Bray-Curtis dissimilarity matrix, were employed to assess the overall dissimilarity in the community structure over the groups of samples (Table I). NPMANOVA tests, for the difference between means or centroids, were performed by comparing the variability 
within groups versus the variability among groups, for data presenting many response variables (i.e. OTUs at $3 \%$ distance) without assuming a multivariate normal distribution (Anderson 2001). Diversity metrics calculation, ordination and statistical analysis were performed with the R 'vegan' package (Oksanen et al. 2013).

Microbiota composition among the groups of Ae. aegypti females was compared by performing (a) a neighbor-joining tree with representative 16S rRNA gene sequences of bacterial taxa (families or genera), coupled with presence/absence data (Letunic \& Bork 2011), (b) a Venn diagram, and (c) an abundance statistical comparison between taxa exhibiting $>2 \%$ relative abundance using the Kruskal-Wallis test, followed by pairwise comparisons with the Dunn's test, if necessary. P-values were adjusted by controlling the false discovery rate (FDR) using the Benjamini-Hochberg procedure. Additionally, relative abundance was also compared among sugar-fed (SFY, SFO) and blood-fed samples (BFY, MRR) (Table I). P-values were adjusted by the Bonferroni method. We used a level of significance for corrected $p$-values $<$ 0.05 . All statistical analyses were performed in R environment (R Development Core Team 2015).

Prediction of potential microbial function - In order to assess whether the diversity changes were associated with differences in the functional profile of Ae. aegypti female microbiota, the functional composition of the gut community was predicted with Phylogenetic Investigation of Communities by Reconstruction of Unobserved States (PICRUSt) (Langille et al. 2013) on the online Galaxy interface. Briefly, this bioinformatics approach incorporates a marker gene (16S rRNA gene) and a database of reference genomes to infer, by ancestral state reconstruction, the functional profile of host-associated and environmental communities (Langille et al. 2013). The nearest sequenced taxon index (NSTI) indicates the accuracy of metagenome predictions, accuracy increasing with decreasing NSTI. Our samples had NSTI of $0.02 \pm 0.002$, considered tractable for PICRUSt prediction (Langille et al. 2013). The predicted genes were annotated using the Kyoto Encyclopedia of Genes and Genomes (KEGG) database. Level 2 KEGG Orthology groups, with more than $2 \%$ relative abundance, were compared by the Kruskal-Wallis test followed by pairwise comparisons with the Dunn's test, if necessary, in $R$ environment ( $R$ Development Core Team 2015). P-values were adjusted by controlling the FDR using the Benjamini-Hochberg procedure.

Isolation of midgut bacteria - Bacterial isolation was conducted with the aim to provide an overview of the culturable microbiota of the Vila Valqueire Ae. aegypti population. We selected four samples from each group, except from BFY in which only two samples were available. For the MRR samples, isolation was conducted after three-four days of collection. Isolation was not performed in wild insects due to logistic issues. Soon after the dissection, each sample was diluted 10 -fold and plated on LBA agar and 10-fold diluted tryptone soya agar (TSA). For the ensuing $72 \mathrm{~h}$, bacterial colonies were screened based on morphology and samples of each morphotype were cryopreserved.
Bacterial DNA was extracted by a boiling and freezing procedure. A 16S rRNA gene segment ( $\sim 519$ $\mathrm{bp}$ ), between the V1 and V3 hypervariable regions, was amplified using the $16 \mathrm{~S}$ universal primers (forward 5'-AGAGTTTGATCCTGGCTCAG-3' and reverse 5'-GTATTACCGCGGCTGCTG-3'). This segment allows the identification of a majority of bacterial groups at genus level. For families with highly similar 16S rRNA gene sequence (e.g., Enterobacteriaceae), a larger fragment $(\sim 1,000 \mathrm{bp})$ between the V1 and V5 hypervariable gene regions was sequenced (forward 5'-AGAGTTTGATCCTGGCTCAG-3' and reverse 5'-GTTGCGCTCGTTGCGGGACT-3'). PCR was performed with GoTaq Flexi DNA Polymerase (Promega, Madison, WI, USA) under standard conditions. Amplicons were purified with the Illustra PCR and Gel Band Purification Kit (GE Healthcare, Little Chalfont, BUX, UK) and sequenced using BigDye Terminator v3.1 Cycle Sequencing Kit, in an ABI 3730 DNA sequencer (Applied Biosystems, Foster City, CA, USA).

Bacteria were identified with the Ribosomal Data Project classifier program (https://rdp.cme.msu.edu/classifier, Version 2.10), with a confidence threshold of $80 \%$ at the genus level. All sequences were submitted to GenBank. A neighbor-joining tree was constructed with a representative 16S rRNA gene sequence for each bacterial genus, coupled with presence/absence data (Letunic \& Bork 2011).

\section{RESULTS}

The midgut microbial diversity throughout $A e$. aegypti lifespan was investigated after the release of laboratory reared females into the urban environment. The microbiota of recaptured specimens was contrasted with the microbiota of laboratory females on different diet and of different age groups, as well as with wild insects from natural breeding sites, employing deep sequencing of bacterial 16S rRNA gene and bacterial isolation (Table I).

Bioinformatics processing and diversity metrics Sequences assigned to the bacteria domain varied, from 4,599-16,935, with conditions. Sequence reads were clustered at maximum 3\% distance into 90 non-singleton OTUs. Sequencing coverage for each group was assessed through rarefaction curves. The curves levelled off without reaching a plateau, suggesting that most microbiota OTUs were detected but some possibly remaining undiscovered (Fig. 1).

As the sample size was constrained by the low recapture rate of the mark-release-recapture experiment [we released 1,730 Ae. aegypti and recaptured 67 ( 4\%) of them using backpack aspirators, with $\sim 54 \%$ in the first two days of collection] and by the methodological procedures (only alive females were dissected), we opted to use all the samples in the analysis and standardised the sampling effort to 4,599 reads. Average richness of OTUs per group ranged from 20.75 (SFO) to 24.5 (BFY). Estimated total richness by ACE varied from 25.3 (SFO) to 33.47 (BFY) (Table II). There were no significant differences among groups for either observed or estimated richness. On the other hand, SFO mosquitoes presented significantly higher Shannon index $(z=3.48$, p-value $<0.01)$, inverse Simpson index $(\mathrm{z}=3.15$, $\mathrm{p}$-value $<0.01)$, and evenness $(\mathrm{z}=3.81$, $\mathrm{p}$-value $<0.01)$ than WD mosquitoes (Table II). 


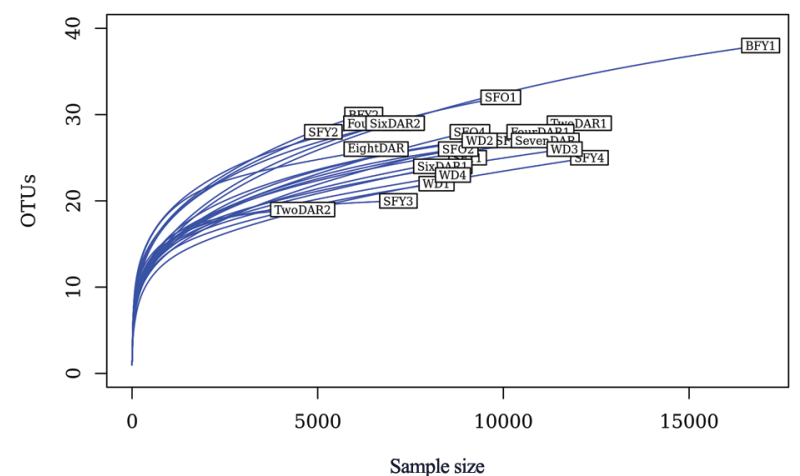

Fig. 1: rarefaction curves for each sample. Operational taxonomic units (OTUs) at 3\% distance were plotted for each sample against the number of sequences generated through 454 pyrosequencing (Sample size). SFY: sugar-fed young; BFY: blood-fed young; SFO: sugar-fed old; DAR: days after release in the mark-release-recapture experiment; WD: wild.

PCoA displayed a single cluster for two SFY samples, BFY, MRR (except TwoDAR1) and WD, whereas there was a higher intra- and intergroup variability in bacterial community structure between the two SFY and SFO samples (Fig. 2). Differences in microbiota community structure among groups were supported by statistical analysis (NPMANOVA: $\mathrm{R}^{2}=0.62$, $\mathrm{p}$-value $<0.01$ ).

Taxonomic assignment and microbiota composition - In total, more than $99 \%$ and $76 \%$ of the reads were assigned to family and genus levels, respectively. Only non-singleton taxa were considered for further analysis. Four bacterial phyla, 23 families and 32 genera were successfully identified in the Ae. aegypti gut microbiota. The bacterial communities colonising female midguts were composed of Proteobacteria (43.7 to $99.9 \%$ ), followed by Bacteroidetes ( 0 to $56.3 \%$ ), Firmicutes ( 0 to $0.3 \%)$ and Actinobacteria ( 0 to $<0.1 \%$ ). Detailed community composition per sample, at family and genus levels, is represented in Fig. 3 and Supplementary Table.

The core microbiota (taxa present in all groups) consisted of 19 out of $41(\sim 46 \%)$ bacterial taxa (Figs $4-5)$,

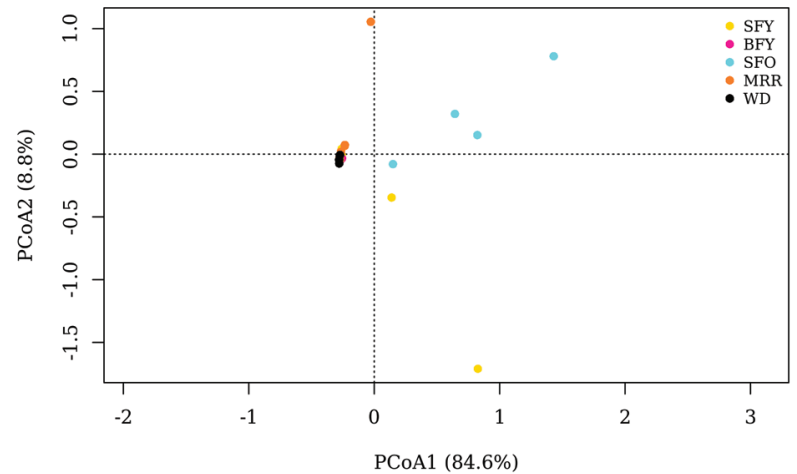

Fig. 2: principal coordinate analysis ( $\mathrm{PCoA})$ of Bray-Curtis distances among Aedes aegypti microbiota samples. Bray-Curtis distance was calculated with operational taxonomic units at 3\% maximum distance. SFY: sugar-fed young (yellow); BFY: blood-fed young (pink); SFO: sugar-fed old (blue); MRR: mark-released-recaptured (orange); WD: wild (black). Each axis shows percentage of variation explained.

comprising 46 to $99 \%$ of microbiota. Pseudomonas was the most abundant genus in the groups, composing up to $70 \%$ of the midgut bacteria in WD females (Fig. 6). The number of taxa per group was 23 in SFY, 24 in BFY, 25 in SFO, 32 in MRR, and 30 in WD mosquitoes (Fig. 4). Together, MRR and WD females contained 11 out of $41(\sim 26 \%)$ taxa present only in these groups, contrasting with four out of 41 taxa $(\sim 9 \%)$ exclusive to SFY, BFY and/or SFO mosquitoes collectively (Figs 4-5).

There were no significant changes in microbiota composition among MRR and BFY, WD, or SFY females, despite the higher abundance of the Elizabethkingia genus in two SFY samples. On the other hand, 38-day-old SFO specimens presented higher abundance of Elizabethkingia (vs. BFY: $\mathrm{z}=-2.44$, p-value $<0.05$; vs. MRR: $\mathrm{z}$ $=-2.91, \mathrm{p}$-value $<0.01 ;$ vs. WD: $\mathrm{z}=2.99$, p-value $<0.05$ ) and Asaia (vs. SFY: z $=3.01$, p-value $<0.05$; vs. BFY: $\mathrm{z}$ $=-2.46$, p-value $<0.05$; vs. MRR: $\mathrm{z}=-2.58$, p-value $<$ 0.05 ; vs. WD: $z=3.01$, p-value $<0.01$ ). Furthermore, the microbiota of SFO insects displayed lower abundance of Pseudomonas (vs. WD: $\mathrm{z}=-2.88$, $\mathrm{p}$-value $<0.05$ ), Oxalo-

\section{TABLE II}

Average \pm standard deviation of diversity metrics calculated for the bacterial communities associated with the analysed Aedes aegypti groups

\begin{tabular}{lccccc}
\hline Group & S & ACE & H' & 1/D & J' \\
\hline SFY & $21.25 \pm 3.20$ & $27.21 \pm 6.2$ & $1.29 \pm 0.21$ & $2.46 \pm 0.51$ & $0.42 \pm 0.05$ \\
BFY & $24.50 \pm 2.12$ & $33.47 \pm 2.16$ & $1.34 \pm 0.17$ & $2.38 \pm 0.36$ & $0.42 \pm 0.04$ \\
SFO & $20.75 \pm 0.96$ & $25.30 \pm 1.89$ & $1.58 \pm 0.10$ & $3.66 \pm 0.69$ & $0.52 \pm 0.03$ \\
MRR & $22.25 \pm 3.92$ & $27.55 \pm 6.75$ & $1.32 \pm 0.13$ & $2.40 \pm 0.30$ & $0.43 \pm 0.03$ \\
WD & $21.25 \pm 1.26$ & $29.88 \pm 5.03$ & $1.14 \pm 0.05$ & $2.10 \pm 0.10$ & $0.37 \pm 0.02$ \\
\hline
\end{tabular}

S: richness (number of operational taxonomic units); ACE: abundance-based coverage estimator; H': Shannon index; 1/D: inverse Simpson index; J': evenness; SFY: sugar-fed young; BFY: blood-fed young; SFO: sugar-fed old; MRR: markreleased-recaptured; WD: wild. 

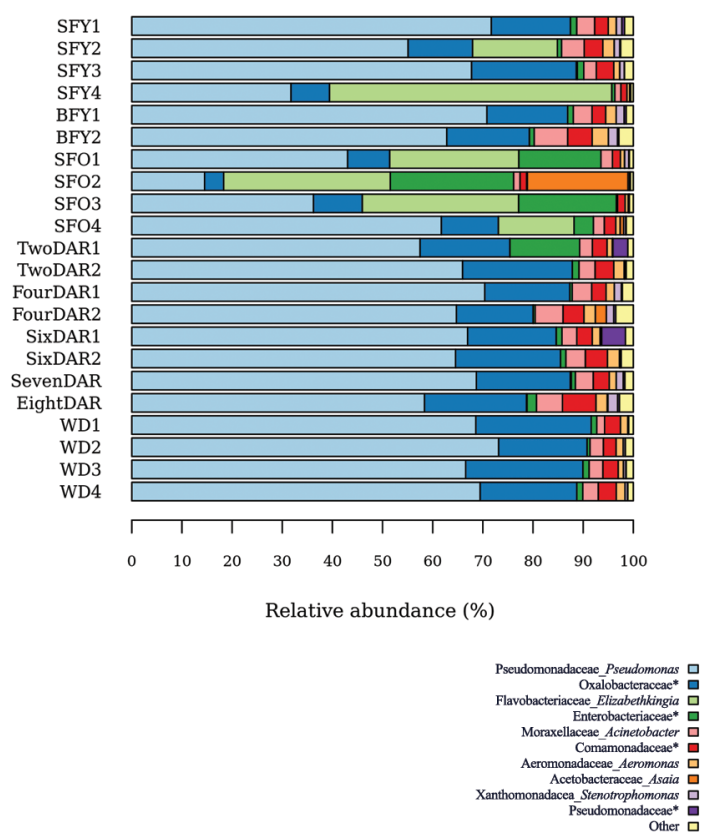

Fig. 3: Aedes aegypti microbiota composition per sample. Gut bacterial taxonomic composition of Ae. aegypti adult females was determined via 16S rRNA gene deep sequencing. SFY: sugar-fed young; BFY: blood-fed young; SFO: sugar-fed old; DAR: days after release in the mark-release-recapture experiment; WD: wild. Taxa with $<2 \%$ relative abundance were pooled as "Other"; *: not discriminated at genus level. bacteraceae (vs. MRR: $\mathrm{z}=2.82$, p-value < 0.05; vs. WD: $\mathrm{z}=-3.15$, p-value $<0.01$ ), Acinetobacter (vs. BFY: $\mathrm{z}=$ 2.71, p-value $<0.05$; vs. MRR: $z=2.89$, p-value $<0.05$ ), Comamonadaceae (vs. MRR: $\mathrm{z}=2.98, \mathrm{p}$-value $<0.05$ ), and Aeromonas (vs. BFY: $\mathrm{z}=2.84$, p-value $<0.05$; vs. MRR: $z=2.84$, p-value $<0.05$ ) (Fig. 6). Strictly sugarfed mosquitoes (SFY, SFO) exhibited significantly higher number of Elizabethkingia than blood-fed mosquitoes $(B F Y, M R R)$ in their microbiota (chi-squared $=10.1$, degrees of freedom $=1$, p-value $<0.05$ ).

Potential microbial function - SFO Ae. aegypti demonstrated significant differences in microbiota composition when compared to BFY, MRR and WD Ae. aegypti females, with an increase in Elizabethkingia and Asaia and a reduction in Oxalobacteraceae, Acinetobacter, and Aeromonas bacteria. Using PICRUSt to make exploratory inferences of the microbial function, we encountered 13 gene families predicted to be differentially represented in SFO in relation to WD females ( $p$-value $<0.05$ ). In addition, two of these predicted functions also showed statistically significant differences between SFO and MRR females (p-value $<0.05$; Fig. 7).

Diversity of culturable bacteria - The culturable midgut microbiota was represented by 39 bacterial colonies, corresponding to 24 morphotypes. Most morphotypes were isolated from both LBA and TSA media (Table III). The identification to the genus level was based on the 16S

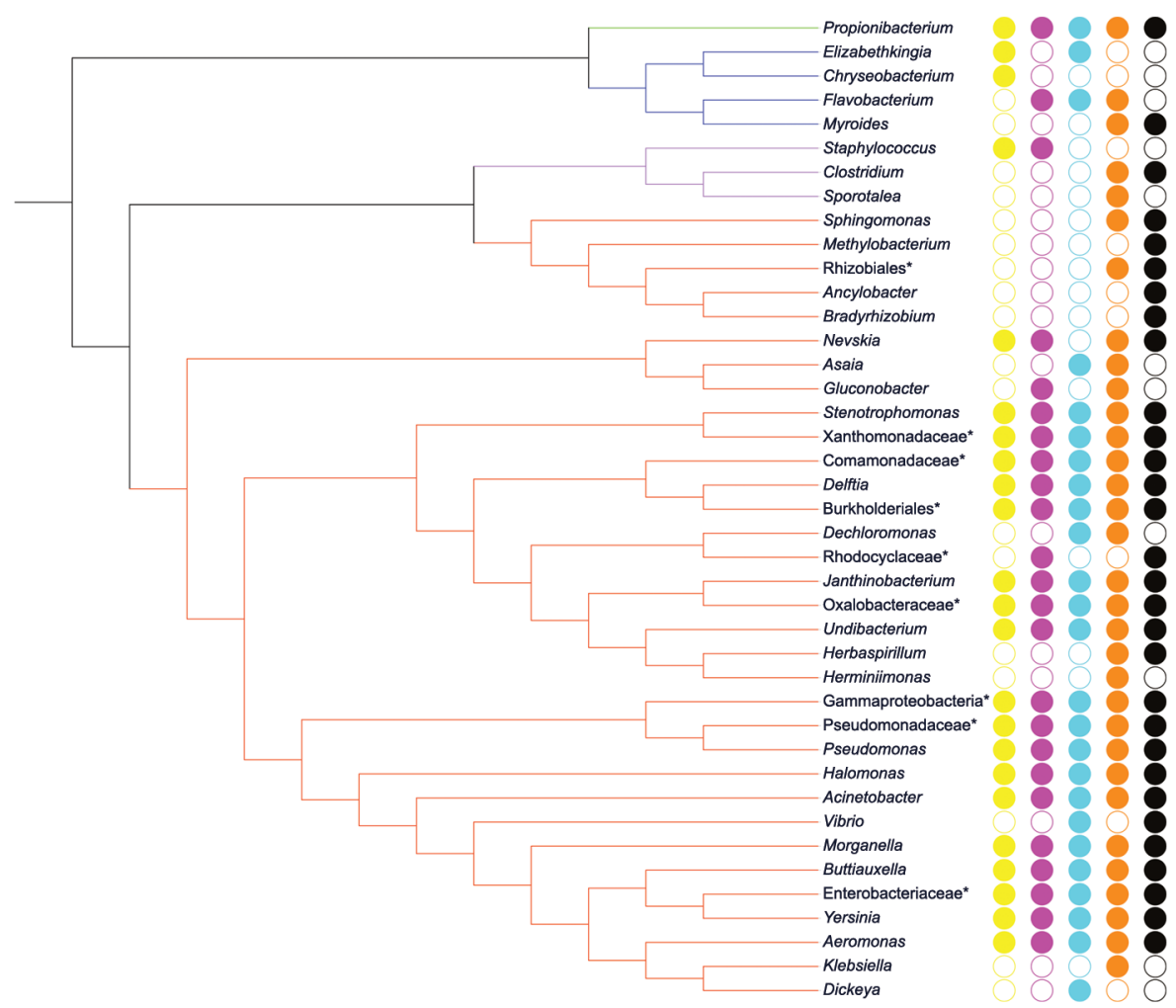

Fig. 4: phylogenetic tree of bacterial taxa detected in Aedes aegypti female midgut via 16S rRNA gene deep sequencing. Yellow circles indicate taxa identified in sugar-fed young females (SFY); pink circles in blood-fed young (BFY); light blue in sugar-fed old (SFO); orange in markreleased-recaptured (MRR); and black in wild (WD) females. Taxa were classified according to their phyla: Bacteroidetes (blue), Actinobacteria (green), Firmicutes (purple), and Proteobacteria (red); *: not discriminated at genus level. 


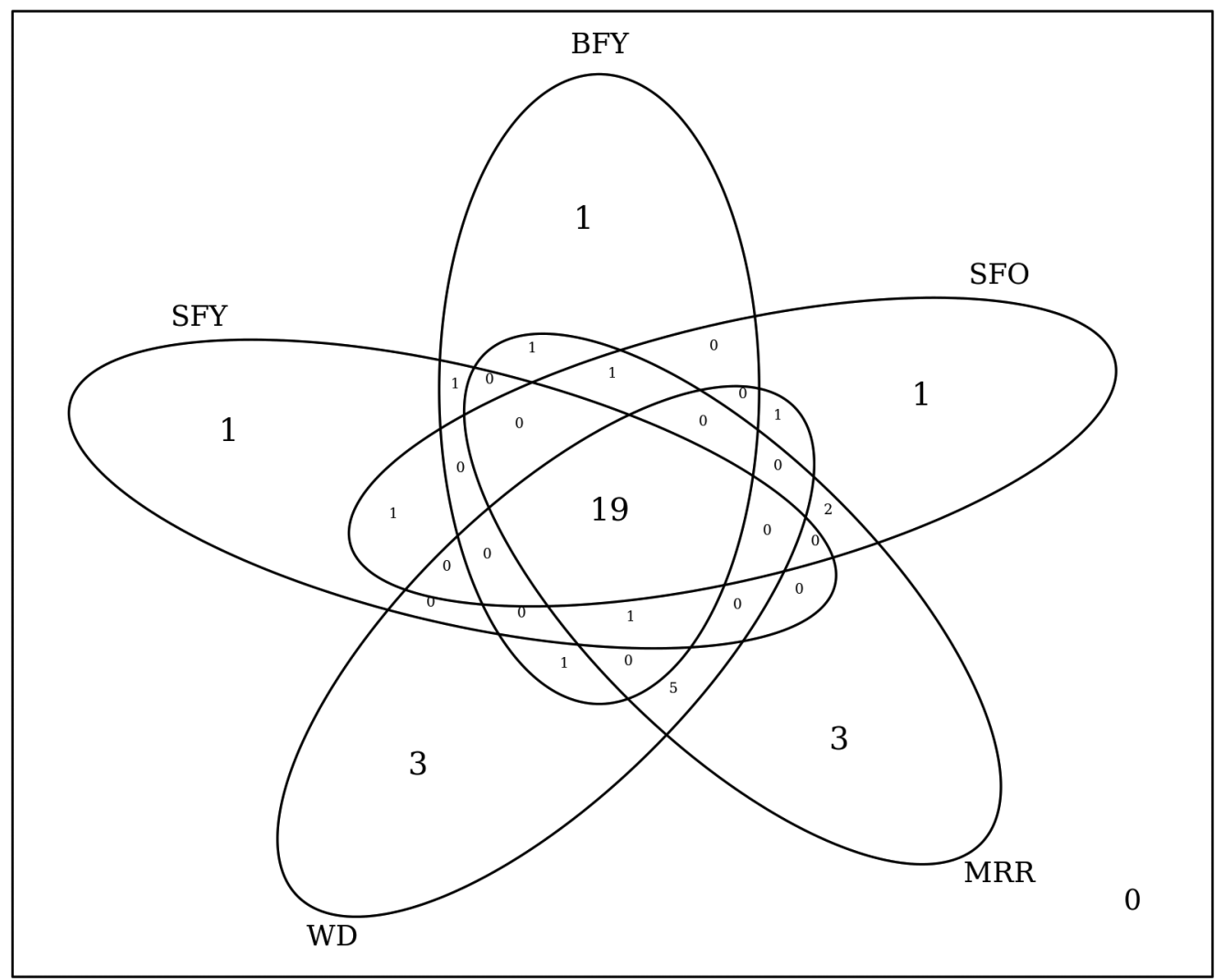

Fig. 5: Venn diagram showing the number of bacterial taxa identified in the Aedes aegypti microbiota via 16S rRNA gene deep sequencing. SFY: sugar-fed young; BFY: blood-fed young; SFO: sugar-fed old; MRR: mark-released-recaptured; WD: wild.

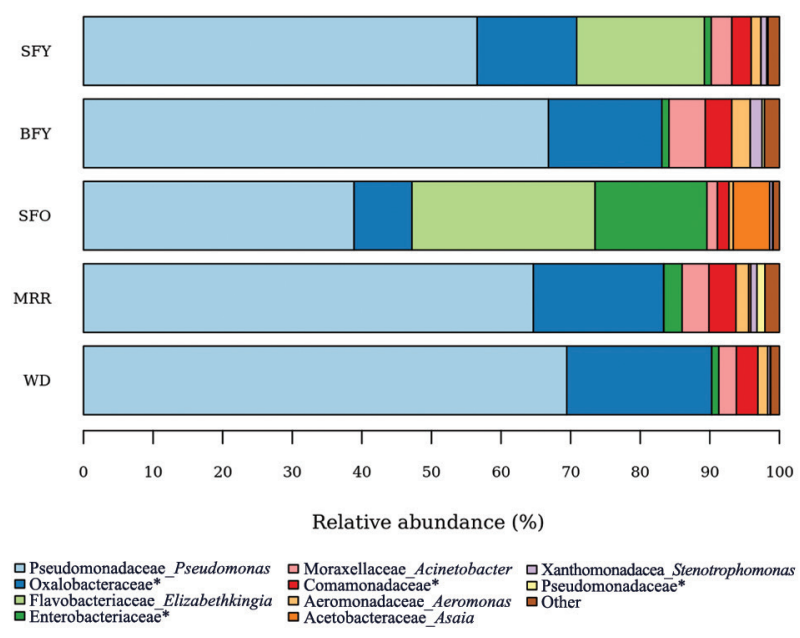

Fig. 6: Aedes aegypti microbiota composition per group. Gut bacterial taxonomic composition of Ae. aegypti adult females was determined via 16S rRNA gene deep sequencing. SFY: sugar-fed young; BFY: blood-fed young; SFO: sugar-fed old; MRR: mark-released-recaptured; WD: wild. Taxa with $<2 \%$ relative abundance were pooled as "Other"; *: not discriminated at genus level.

rRNA gene sequence. We recovered bacteria belonging to three phyla, eight families and nine genera (Table III, Fig. 8). The majority of genera belonged to the Proteobac- teria phylum (Fig. 8). Elizabethkingia and Enterobacter were recovered from SFY group, whereas only Pseudomonas was isolated from BFY group. SFO females still carried Elizabethkingia and Enterobacter 35 days later, but Serratia was also detected in the tested specimens. An expressive change was observed in the culturable microbiota composition after release, as MRR females presented Asaia, Azorhizobium, Microbacterium, Pseudomonas, Paracoccus (FourDAR) and Herbaspirillum (EightDAR), whereas Enterobacter and Elizabethkingia were no longer cultivable. Pseudomonas was the only genus shared between blood-fed mosquitoes, from both MRR and laboratory (BFY) (Table III, Fig. 8).

\section{DISCUSSION}

The midgut microbiota of mosquitoes is supposedly a function of host intrinsic factors (e.g., genetics), feeding behaviour and environment (e.g., climate, larval habitats and vertebrate hosts) (Zouache et al. 2011, Osei-Poku et al. 2012, Hegde et al. 2015). Nevertheless, gut community ecology remain poorly investigated from the standpoint of mosquito interactions within its natural environment. Therefore, the present study assessed the diversity and the dynamics of Ae. aegypti microbiota under natural conditions, using the mark-release-recapture experiment. Herein, we offer evidence for Ae. aegypti female lifelong core microbiota, i.e. the community composition comprising the majority of microbiota was stable after 


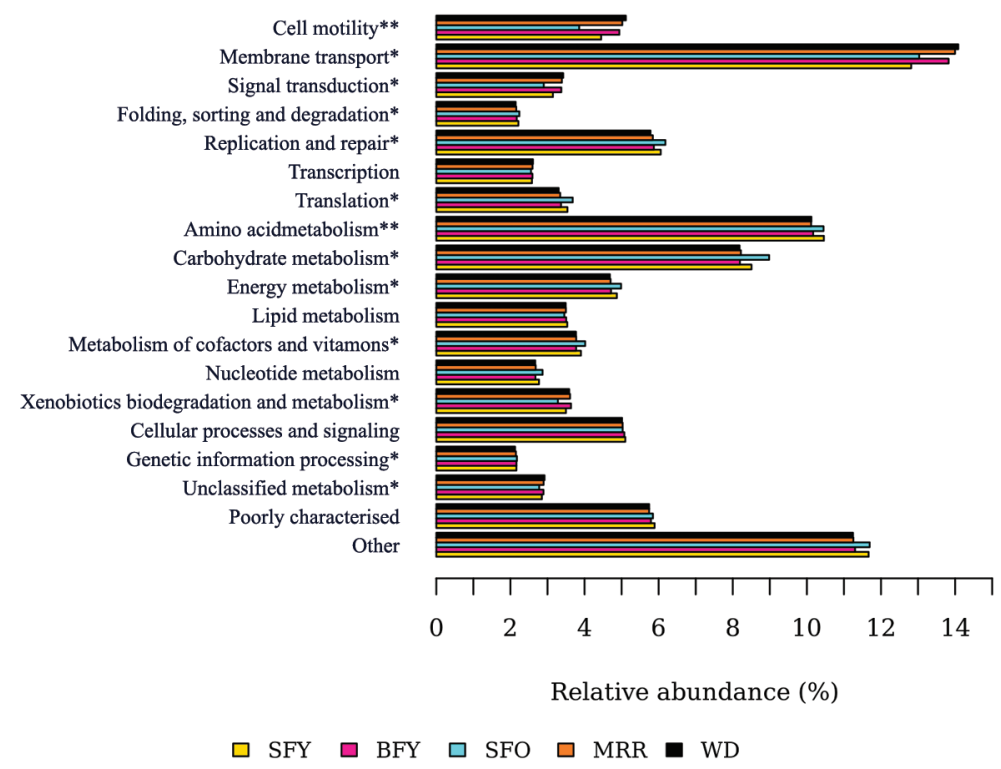

Fig. 7: predicted functions of the bacterial communities found in Aedes aegypti adult females. Yellow bars indicate Kyoto Encyclopedia of Genes and Genomes metabolic pathways predicted in the microbiota of sugar-fed young females (SFY); pink bars in blood-fed young (BFY); light blue in sugar-fed old (SFO); orange in mark-released-recaptured (MRR); and black in wild females (WD). *: indicates gene categories significantly different between SFO and WD mosquitoes; **: indicates gene categories significantly different among SFO and both MRR and WD mosquitoes (Kruskal-Wallis test, Benjamini-Hochberg corrected p-value $<0.05$ ).

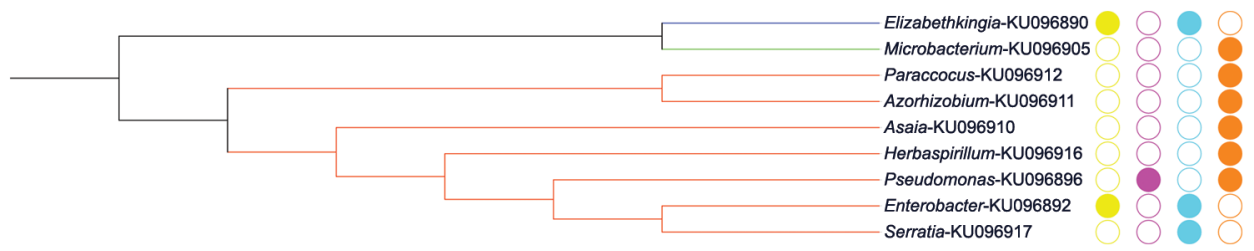

Fig. 8: phylogenetic tree of culturable bacterial genera recovered from Aedes aegypti females. SFY: sugar-fed young (yellow); BFY: blood-fed young (pink); SFO: sugar-fed old (blue); MRR: mark-released-recaptured (orange). Bacterial genera were classified according to their phyla: Bacteroidetes (blue), Actinobacteria (green) and Proteobacteria (red).

mosquitoes were released into the urban settlement. Additionally, we show that the female dietary regime and mosquito age were associated with the abundance of some bacterial groups in the Ae. aegypti microbiota.

After release, the Ae. aegypti females presumably blood-fed on a range of vertebrate hosts with diet supplements of different carbohydrate sources (fruits and flowers), which could represent potential sources of bacteria acquisition during their adult life. In contrast to the blood meals, the sugar meals do not go directly to the midgut, but are stored in the crop as food reserve (Clements 1992). This structure harbours bacteria, such as Bacillus, Serratia and Pichia (Gusmão et al. 2007), which have also been detected in the midgut (Gusmão et al. 2010). Therefore, it is suggested that the bacteria acquired from sugar sources can be transferred to the midgut along with food, when convenient (Gusmão et al. 2007).

For $>2 \%$ abundant bacteria, MRR female microbiota composition was not different from the specimens main- tained in the laboratory under SFY or BFY regimes, despite of two SFY samples presenting high levels of the Elizabethkingia genus. In addition, microbiota was similar between laboratory reared (SFY, BFY and MRR) and WD mosquitoes, regardless of the differences between conditions in laboratory and natural breeding sites, i.e. water biogeochemical characteristics, microclimate and food type. This relatively constant midgut community composition in adult mosquitoes may reflect a competitive environment (Terenius et al. 2012) and is of great significance for the application of midgut microbiota in disease control approaches, as association of bacteria with host must be steady over the host adult lifetime (Capone et al. 2013).

MRR females exclusively shared $<2 \%$ abundant bacteria taxa with WD insects suggesting bacterial acquisition in the natural habitat after release. SFO females, which remained in the laboratory for 35 days, displayed fewer differences in microbiota composition in relation to SFY and BFY mosquitoes, indicating that this appar- 


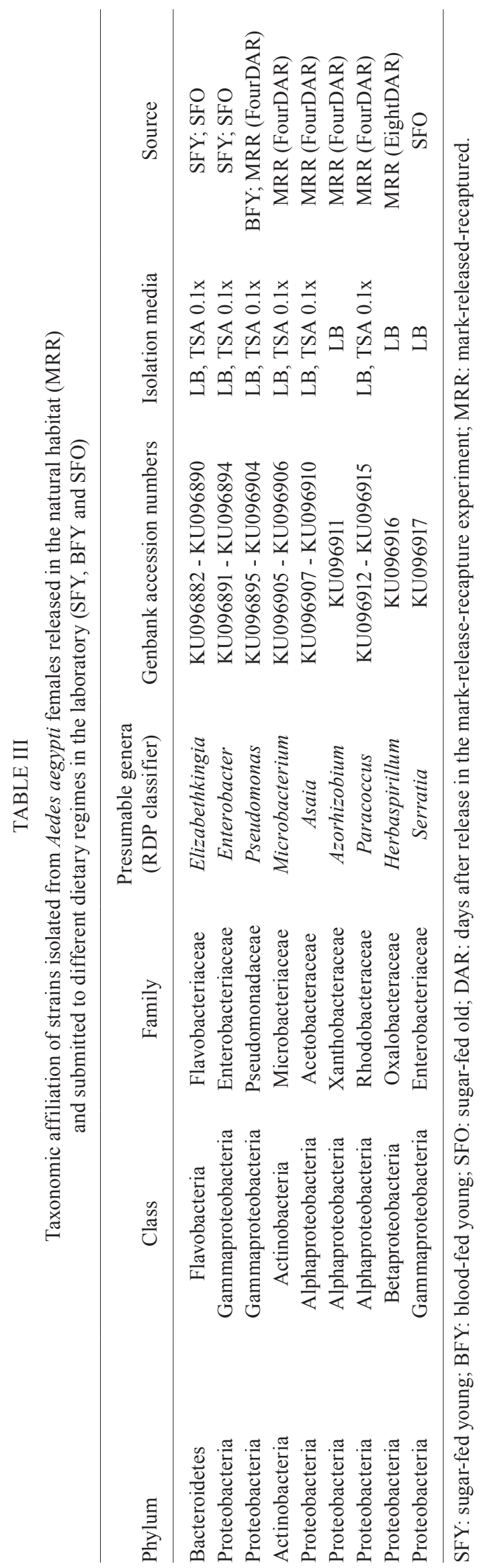

ent midgut bacterial acquisition did not take place at the same magnitude within the laboratory environment. In general, MRR female microbiota was structurally more similar to WD and BFY than to SFY and SFO midgut communities (Fig. 2), suggesting that the environment and the feeding behaviour can synergistically modulate the presence of some bacterial groups.

Sugar-fed females (SFY and SFO) harboured the Elizabethkingia (Flavobacteriaceae) genus, which was absent or undetectable in blood-fed (BFY and MRR) and WD specimens. A reduction in Elizabethkingia was observed in Anopheles gambiae after blood feeding, applying the same deep sequencing approach (Wang et al. 2011). We recovered Serratia from sugar-fed (SFO) females, whereas a previous study reported this genus was dominant in the Ae. aegypti midgut after sugar feeding and during the whole blood digestion (Gusmão et al. 2010). Asaia was previously isolated from sugar-fed $A e$. aegypti and upon initial blood digestion (Gusmão et al. 2010), whereas we recovered this genus in blood-fed MRR mosquitoes four days after release. Thus, strains isolated from distinct mosquito populations might differ in their growth characteristics inside the midgut environment and/or in the isolation media.

Contrasting the stability of microbiota composition in the first week after adult emergence, the microbiota of SFO females revealed a remarkable increase in Elizabethkingia and, to a lesser extent, Asaia (Acetobacteraceae). In addition, significant functional and metabolic alterations were predicted in the microbiota of SFO versus WD and MRR females. Microbial community shifts over time could be related to the mosquito immune capacity to control proliferation of certain bacteria (Hillyer et al. 2005), nutrient availability in the gut environment (Montagna et al. 2015) and/or result of direct interaction among colonising bacteria, e.g. competition (Dong et al. 2009).

The 16S rRNA gene sequences (1,020 bp) from Elizabethkingia strains recovered in this study share $99 \%$ identity with the Che01 strain (GenBank accession JX067927) isolated from the midgut of Anopheles stephensi. The ethyl acetate and acetone extracts produced by the Che 01 strain showed antibacterial, antifungal and antiplasmodial activities, which might explain the dominance of this bacteria in the microbiota of An. stephensi (Ngwa et al. 2013). Similarly, Asaia have been shown to inhibit the colonisation and maternal transmission of the intracellular bacteria Wolbachia (Hughes et al. 2014). Further investigation is required to determine whether the Ae. aegypti microbiota strains possess similar capabilities.

Studies on the microbiota of Ae. aegypti females recovered Asaia, Elizabethkingia, Enterobacter, Pseudomonas and Serratia genera from laboratory colonies (Gusmão et al. 2010, Terenius et al. 2012) as well as wild populations from Panama (Ramírez et al. 2012). Additionally, $50 \%$ of the genera detected through deep sequencing were also found in Ae. aegypti from Kenya, applying the same approach (Osei-Poku et al. 2012). The overlapping microbiota composition among Ae. aegypti populations from distinct geographic regions suggest a core microbiota stably associated with this species, acquired through mechanisms of vertical transmission and/or constant en- 
vironmental exposure (Zouache et al. 2011). Ae. aegypti breeds almost exclusively in domestic containers recurrent across the globe, such as discarded tires and plastic buckets, in which several of this bacteria taxa have been detected (Ponnusamy et al. 2008, Dada et al. 2014). This could partially explain the similarities found in the microbiota of geographically distant populations.

The present study shows that (i) the dominant bacteria were ubiquitous in the female mosquito midgut during the first week after emergence; this core microbiota was independent of rearing conditions (laboratory or natural breeding site) and diet regime (sugar or blood) after being released into the natural habitat, where females fed on blood and carbohydrate sources; (ii) the Elizabethkingia genera was associated with sugar-fed females; (iii) the microbiota structure changed in older mosquitoes; (iv) the shifts in the microbiota of older Ae. aegypti females were predicted to influence the functional and metabolic profile of the midgut community; and (v) the microbiota identified during this study shared many bacterial taxa with Ae. aegypti from distinct geographic locations, suggesting a core microbiota associated with this mosquito species. Investigation of the factors influencing microbiota dynamic in Ae. aegypti provides insight into the impacts of bacterial community on mosquito biology and evolution, which can be explored for the development of new disease control tools.

\section{ACKNOWLEDGEMENTS}

To Roberto Costa Peres, for field assistance, Fernanda Freitas and Érica Miranda, for providing technical support for bacterial isolation and identification, Michel Marín, for bioinformatics support, and to the Oswaldo Cruz Institute highthroughput sequencing platform.

\section{REFERENCES}

Anderson MJ. A new method for non-parametric multivariate analysis of variance. Austral Ecol. 2001; 26: 32-46.

Capone A, Ricci I, Damiani C, Mosca M, Rossi P, Scuppa P, et al. Interactions between Asaia, Plasmodium and Anopheles: new insights into mosquito symbiosis and implications in malaria symbiotic control. Parasit Vectors. 2013; 6(1): 182.

Clements AN. The biology of mosquitoes: development, nutrition and reproduction. 1st ed. Wallingford: CABI Publishing; 1992.

Dada N, Jumas-Bilak E, Manguin S, Seidu R, Stenström TA, Overgaard HJ. Comparative assessment of the bacterial communities associated with Aedes aegypti larvae and water from domestic water storage containers. Parasit Vectors. 2014; 7: 391.

Dong Y, Manfredini F, Dimopoulos G. Implication of the mosquito midgut microbiota in the defense against malaria parasites. PLoS Pathog. 2009; 5(5): e1000423.

Dutra HL, dos Santos LM, Caragata EP, Silva JB, Villela DA, Macielde-Freitas R, et al. From lab to field: the influence of urban landscapes on the invasive potential of Wolbachia in Brazilian Aedes aegypti mosquitoes. PLoS Negl Trop Dis. 2015; 9(4): e0003689.

Edgar RC, Haas BJ, Clemente JC, Quince C, Knight R. UCHIME improves sensitivity and speed of chimera detection. Bioinformatics. 2011; 27(16): 2194-200.

Gusmão DS, Santos AV, Marini DC, Bacci Jr M, Berbert-Molina MA, Lemos FJ. Culture-dependent and culture-independent characterization of microorganisms associated with Aedes aegypti
(Diptera: Culicidae) (L.) and dynamics of bacterial colonization in the midgut. Acta Trop. 2010; 115: 275-81.

Gusmão DS, Santos AV, Marini DC, Russo ES, Peixoto AMD, Bacci $\mathrm{Jr} \mathrm{M}$, et al. First isolation of microorganisms from the gut diverticulum of Aedes aegypti (Diptera: Culicidae): new perspectives for an insect-bacteria association. Mem Inst Oswaldo Cruz. 2007; 102(8): 919-24.

Harrington LC, Scott TW, Lerdthusnee K, Coleman RC, Costero A, Clark GG, et al. Dispersal of the dengue vector Aedes aegypti within and between rural communities. Am J Trop Med Hyg. 2005; 72(2): 209-20.

Hegde S, Rasgon JL, Hughes GL. The microbiome modulates arbovirus transmission in mosquitoes. Curr Opin Virol. 2015; 15: 97-102.

Hillyer JF, Schmidt SL, Fuchs JF, Boyle JP, Christensen BM. Ageassociated mortality in immune challenged mosquitoes (Aedes aegypti) correlates with a decrease in haemocyte numbers. Cell Microbiol. 2005; 7(1): 39-51.

Hughes GL, Dodson BL, Johnson RM, Murdock CC, Tsujimoto H, Suzuki Y, et al. Native microbiome impedes vertical transmission of Wolbachia in Anopheles mosquitoes. Proc Natl Acad Sci USA. 2014; 111(34): 12498-503.

Kim CH, Lampman LR, Muturi EJ. Bacterial communities and midgut microbiota associated with mosquito populations from waste tires in East-Central Illinois. J Med Entomol. 2015; 52(1): 63-75.

Langille MG, Zaneveld J, Caporaso JG, McDonald D, Knights D, Reyes JA, et al. Predictive functional profiling of microbial communities using 16S rRNA marker gene sequences. Nat Biotechnol. 2013; 31(9): 814-21.

Letunic I, Bork P. Interactive tree of life v2: online annotation and display of phylogenetic trees made easy. Nucleic Acids Res. 2011; 39(Web Server issue): W475-8.

Maciel-de-Freitas R, Codeço CT, Lourenço-de-Oliveira R. Daily survival rates and dispersal of Aedes aegypti females in Rio de Janeiro, Brazil. Am J Trop Med Hyg. 2007; 76(4): 659-65.

McDonald PT. Population characteristics of domestic Aedes aegypti (diptera: culicidae) in villages on the Kenya coast. II. Dispersal within and between villages. J Med Entomol. 1977; 14(1): 49-53.

Minard G, Mavingui P, Moro CV. Diversity and function of bacterial microbiota in the mosquito holobiont. Parasit Vectors. 2013; 6: 146.

Minard G, Tran FH, Van VT, Goubert C, Bellet C, Lambert G, et al. French invasive Asian tiger mosquito populations harbor reduced bacterial microbiota and genetic diversity compared to Vietnamese autochthonous relatives. Front Microbiol. 2015; 6: 970.

Montagna M, Chouaia B, Mazza G, Prosdocimi EM, Crotti E, Mereghetti V, et al. Effects of the diet on the microbiota of the red palm weevil (Coleoptera: Dryophthoridae). PLoS ONE. 2015; 10(1): e0117439.

Ngwa CJ, Glöckner V, Abdelmohsen UR, Scheuermayer M, Fischer $\mathrm{R}$, Hentschel U, et al. 16S rRNA gene-based identification of Elizabethkingia meningoseptica (Flavobacteriales: Flavobacteriaceae) as a dominant midgut bacterium of the Asian malaria vector Anopheles stephensi (Dipteria: Culicidae) with antimicrobial activities. J Med Entomol. 2013; 50(2): 404-14.

Oksanen J, Blanchet FG, Kindt R, Legendre P, Minchin PR, O'Hara $\mathrm{RB}$, et al. Vegan: community ecology package. $\mathrm{R}$ package version 2.1-26/r2441 [software]. 2013 [cited 2016 Jun 28]. Available from: https://CRAN.R-project.org/package=vegan.

Osei-Poku J, Mbogo CM, Palmer WJ, Jiggins FM. Deep sequencing reveals extensive variation in the gut microbiota of wild mosquitoes from Kenya. Mol Ecol. 2012; 21(20): 5138-50. 
Ponnusamy L, Xu N, Stav G, Wesson DM, Schal C, Apperson CS. Diversity of bacterial communities in container habitats of mosquitoes. Microb Ecol. 2008; 56(4): 593-603.

R Development Core Team. R: a language and environment for statistical computing. $\mathrm{R}$ foundation for statistical computing. Version 3.2.3 [software]. 2015 [cited 2016 Jun 28]. Available from: https:// www.R-project.org/.

Ramírez JL, Short SM, Bahia AC, Saraiva RG, Dong Y, Kang S, et al. Chromobacterium Csp_P reduces malaria and dengue infection in vector mosquitoes and has entomopathogenic and in vitro antipathogen activities. PLoS Pathog. 2014; 10(10): e1004398.

Ramírez JL, Souza-Neto J, Cosme RT, Rovira J, Ortiz A, Pascale JM, et al. Reciprocal tripartite interactions between the Aedes aegypti midgut microbiota, innate immune system and dengue virus influences vector competence. PLoS Negl Trop Dis. 2012; 6(3): e1561.

Rosenberg ZI, Rosenberg E. Role of microorganisms in the evolution of animals and plants: the hologenome theory of evolution. FEMS Microbiol Rev. 2008; 32(5): 723-35.

Schloss PD, Westcott SL, Ryabin T, Hall JR, Hartmann M, Hollister $\mathrm{EB}$, et al. Introducing mothur: open-source, platform-independent, community-supported software for describing and comparing microbial communities. Appl Environ Microbiol. 2009; 75(23): 7537-41
Terenius O, Lindh JM, Eriksson-Gonzales K, Bussière L, Laugen AT, Bergquist $\mathrm{H}$, et al. Midgut bacterial dynamics in Aedes aegypti. FEMS Microbiol Ecol. 2012; 80(3): 556-65.

Villegas LM, Pimenta PFP. Metagenomics, paratransgenesis and the Anopheles microbiome: a portrait of the geographical distribution of the anopheline microbiota based on a meta-analysis of reported taxa. Mem Inst Oswaldo Cruz. 2014; 109(5): 672-84.

Wang Q, Garrity GM, Tiedje JM, Cole JR. Naive Bayesian classifier for rapid assignment of rRNA sequences into the new bacterial taxonomy. Appl Environ Microbiol. 2007; 73(16): 5261-7.

Wang Y, Gilbreath TM 3rd, Kukutla P, Yan G, Xu J. Dynamic gut microbiome across life history of the malaria mosquito Anopheles gambiae in Kenya. 2011; 6(9): e24767.

Xi Z, Ramírez JL, Dimopoulos G. The Aedes aegypti toll pathway controls dengue virus infection. PLoS Pathog. 2008; 4(7): e1000098.

Zouache K, Michelland RJ, Failloux AB, Grundmann GL, Mavingui P. Chikungunya virus impacts the diversity of symbiotic bacteria in mosquito vector. Mol Ecol. 2012; 21(9): 2297-309.

Zouache K, Raharimalala FN, Raquin V, Tran-Van V, Raveloson LH, Ravelonandro P, et al. Bacterial diversity of field-caught mosquitoes, Aedes albopictus and Aedes aegypti, from different geographic regions of Madagascar. FEMS Microbiol Ecol. 2011; 75(3): 377-89. 